\title{
Design of a Large Helical System for Magnetic Fusion Experiments
}

\author{
By P. R. Garabedian \\ Dedicated to Eugene Isaacson on the occasion of his 70 th birthday
}

\begin{abstract}
Computer codes are described that solve the partial differential equations of ideal magnetohydrodynamics describing toroidal equilibrium of a plasma. These numerical methods are applied to find a helical coil winding law for stellarator experiments designed so that the parallel current is relatively low.
\end{abstract}

1. The Computational Model. The availability of supercomputers has resulted in wide acceptance of three-dimensional codes as a mathematical tool to assess configurations for the magnetic confinement of a plasma to generate energy by nuclear fusion. The primary codes are based on a variational principle of ideal magnetohydrodynamics that leads to iterative schemes to solve the partial differential equations describing toroidal equilibrium of the plasma [1]. However, more elementary line tracing routines are helpful in studying the existence of magnetic surfaces. Also there is now adequate computer capacity to estimate transport of both ions and electrons by the Monte Carlo method [2]. Here we shall explain how these techniques of numerical analysis can be used in the design of large helical confinement systems planned for magnetic fusion experiments that may cost hundreds of millions of dollars.

Magnetohydrodynamic equilibria are governed by the system of partial differential equations

$$
\nabla \cdot B=0, \quad J \times B=\nabla p,
$$

where $B$ is the magnetic field, $J=\nabla \times B$ is the current density, and $p$ is the fluid pressure. Solutions are sought for which there are nested toroidal flux surfaces $s=$ const with

$$
B \cdot \nabla s=0, \quad p=p(s) .
$$

The equations of ideal magnetohydrodynamics imply that the plasma is confined by these surfaces. The limit of the increment of the poloidal angle divided by the increment of the toroidal angle over an infinitely long magnetic line tracing out a surface $s=$ const is known as the rotational transform $\iota=\iota(s)$. Subject to appropriate mass and flux constraints on the functions $p$ and $\iota$, equilibrium of the plasma is found to minimize the potential energy

$$
E=\iiint\left[\frac{B^{2}}{2}+\frac{p}{\gamma-1}\right] d x d y d z
$$

Received January 13, 1988.

1980 Mathematics Subject Classification (1985 Revision). Primary 65N05, 76W05. 
where $\gamma$ is the ratio of specific heats. Global stability of the equilibrium can be tested by means of a second minimization of $E$ which leads to a growth rate $\omega$ defined by the Rayleigh quotient

$$
-\omega^{2}=\frac{E_{2}-E_{1}}{\|\xi\|^{2}}
$$

where the denominator is a norm of the displacement $\xi$ of the plasma [1].

Fourier analysis of the parallel current $\lambda=J \cdot B / B^{2}$ in suitable flux coordinates shows that in general a smooth solution of the equilibrium problem swept out by nested flux surfaces $s=$ const does not exist in three dimensions when there is no two-dimensional symmetry [2]. In the harmonic case

$$
\nabla \cdot B=\nabla \times B=0
$$

at zero pressure, line tracing can be used to investigate the magnetic surfaces. Numerical results of $\mathrm{Ng}[3]$ show that good surfaces usually exist on the length scale of the electron gyroradius, so there is little adverse effect on transport. Truncation error tends to produce ergodic regions which may actually look more like fractals of islands in the exact solution. Monte Carlo calculations do indicate, though, that transport is sensitive to islands in the drift surfaces associated with a finite gyroradius, which depend significantly on the electrostatic field producing quasineutrality of the ions and electrons.

A nested surface hypothesis is built into the BETA code for computation of toroidal equilibria [1]. Apparent incompatibility with the above theorem about nonexistence of smooth solutions without two-dimensional symmetry is explained by the observation that the numerical method approximates weak solutions in which magnetic islands are replaced by current sheets. The more precise meaning of a weak solution in nonparametric form is specified in a natural way by the variational conditions for the potential energy. In practice, the Mercier local stability criterion can be invoked as a diagnostic for the presence of islands due to a pressure gradient. Severe violation of that criterion may signify that a so-called soft limit has been reached by the plasma parameter $\beta=2 p / B^{2}$, which can rise no further because of transport related to the islands.

The BETA equilibrium and stability code has been validated through comparison with exact two-dimensional solutions, with other equilibrium codes, and with line tracing calculations [1], [3]. Line tracing itself is a valuable tool in the design of fusion configurations and may be used to determine whether magnetic surfaces depend continuously on coil parameters. Any device that is seriously proposed should be tested for the $\beta$ limit due to nonlinear stability, for breakdown of magnetic surfaces corresponding to violation of the Mercier criterion, for adequate shear $\iota^{\prime}$ and positive magnetic well, for continuous dependence of the KAM surfaces on coil parameters, and for neoclassical transport of both ions and electrons. In the next section we shall discuss these issues for a special example defined by a helical coil winding law that seems to have desirable properties.

2. A Helical Coil Winding Law. The largest magnetic fusion experiments have been tokamaks, which cannot be operated in a steady state because they require net toroidal current to produce a poloidal field confining the plasma. On 
the other hand, stellarators can be operated in a steady state because their poloidal field is obtained from helical coils. However, the geometry is so complicated that computer codes of the kind we have described must be used to analyze equilibrium, stability and transport. In both Germany and Japan there are now plans to build stellarators as big as the present tokamaks. In this section we describe a helical coil winding law for a system that we believe to be optimal for such experiments [2].

The best stellarators seem to be those with multiple harmonics designed to reduce the parallel current $\lambda$ so that the magnetic field becomes relatively insensitive to increments in the pressure parameter $\beta$. Heliacs, which have a pronounced crescent in the shape of the plasma and a marked helical excursion of the magnetic axis, are also promising, especially when the magnetic field is almost two-dimensional. Recently, Nuehrenberg and Zille [4] have introduced the Helias configuration, which may be viewed as a Heliac of moderate aspect ratio that is provided with sidebands reducing the parallel current so as to meet the Mercier local stability criterion. Since most of the previous stellarator experiments, including the Heliotron $\mathrm{E}$ at Kyoto University, have had two helical coils, we have sought an $l=2$ winding law of that kind which would yield a configuration with most of the desirable properties of the Helias. This has been accomplished by combining $l=1$ and $l=2$ harmonics $[2]$.

First the BETA code has been used to find an equilibrium with high critical $\beta$ and long confinement time $\tau$ that is defined by a Fourier series for the plasma boundary having just a few well-chosen coefficients. Then a new line tracing routine has been introduced to determine coils from the Biot-Savart law

$$
B=I \oint \nabla \frac{1}{R} \times(d x, d y, d z)
$$

so as to generate magnetic surfaces close to the shape prescribed by the equilibrium. We have had partial success in minimizing the distance of a magnetic line from the given surface, although that is not a well-posed problem because the coils must not come too near to the plasma. The main difficulty is to achieve an adequate crescent in the outermost cross section of the torus while simultaneously creating sidebands that reduce the parallel current $\lambda$. What succeeds is an appropriate choice of the parameters appearing in an analytical representation

$$
r+i z=A_{k}+D_{k} \exp i\left(\theta-\theta_{k}+\sum C_{k m} \sin m \theta\right)
$$

of the two helical coils. In this construction it has been helpful to iterate back and forth between runs of the equilibrium and line tracing codes so as to arrive at a configuration minimizing $\lambda$. Fourier coefficients of the normal component of the magnetic field on the prescribed surface of the plasma provide a measure of the error in the winding law.

In Figure 1 we display the result of a run of the line tracing code. Four Poincare sections of the magnetic surfaces, together with the prescribed plasma boundary, are shown in one out of eight field periods. Details about the winding law become visible in the plots. There is an elongated outer helical coil that more or less serves as a perfectly conducting wall. In practice, this might be constructed from superconducting material to enhance stability. The inner helical coil is round and generates a poloidal field producing the desired crescent. Toroidal and vertical 

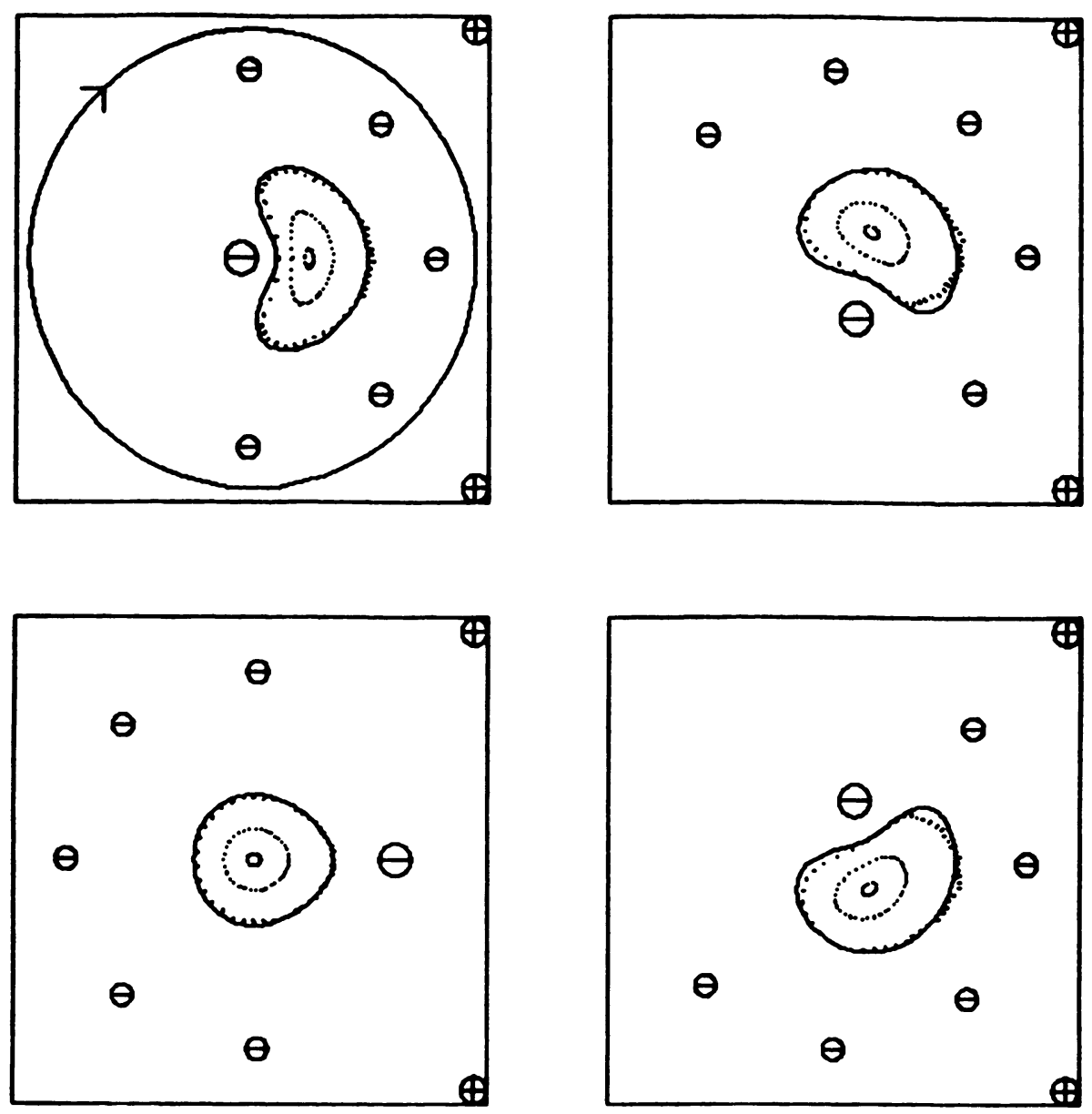

FIGURE 1

Four cross sections of an optimized stellarator configuration showing the comparison of a desired plasma shape with magnetic surfaces traced in the vacuum field of an $l=2$ winding law. The five small circles with minus signs indicate filaments defining an outer helical coil, while the larger circle with a minus sign indicates a poloidal field coil. The circles with plus signs represent vertical field coils, and the very large circle with an arrow in the first plot represents a toroidal field coil.

field coils complete the system, which confines a plasma of aspect ratio nine with rotational transform in the range $0.55<\iota<0.85$. Low parallel current is expected to make the product $\beta \tau$ of the plasma parameter and the confinement time an order of magnitude larger than has been observed in past stellarator experiments.

Figure 2 is a plot of the Rayleigh quotient $-\omega^{2} / m^{2}$ as a function of $\beta$ for an equilibrium related to the one in Figure 1. The rotational transform $\iota$ is just below unity, so resonant modes with the poloidal and toroidal wave numbers $m=n=1$, $m=n=2$ and $m=n=3$ have been tested for global stability [2]. The $\beta$ limit is seen to exceed 0.05 , but there appears to be no second stability region. This is only one example from a family of such configurations, including certain Heliacs, that seem to have attractive properties according to the computational theory we have outlined. The issue is whether the calculations are convincing enough to establish them as candidates for some future experiment. 


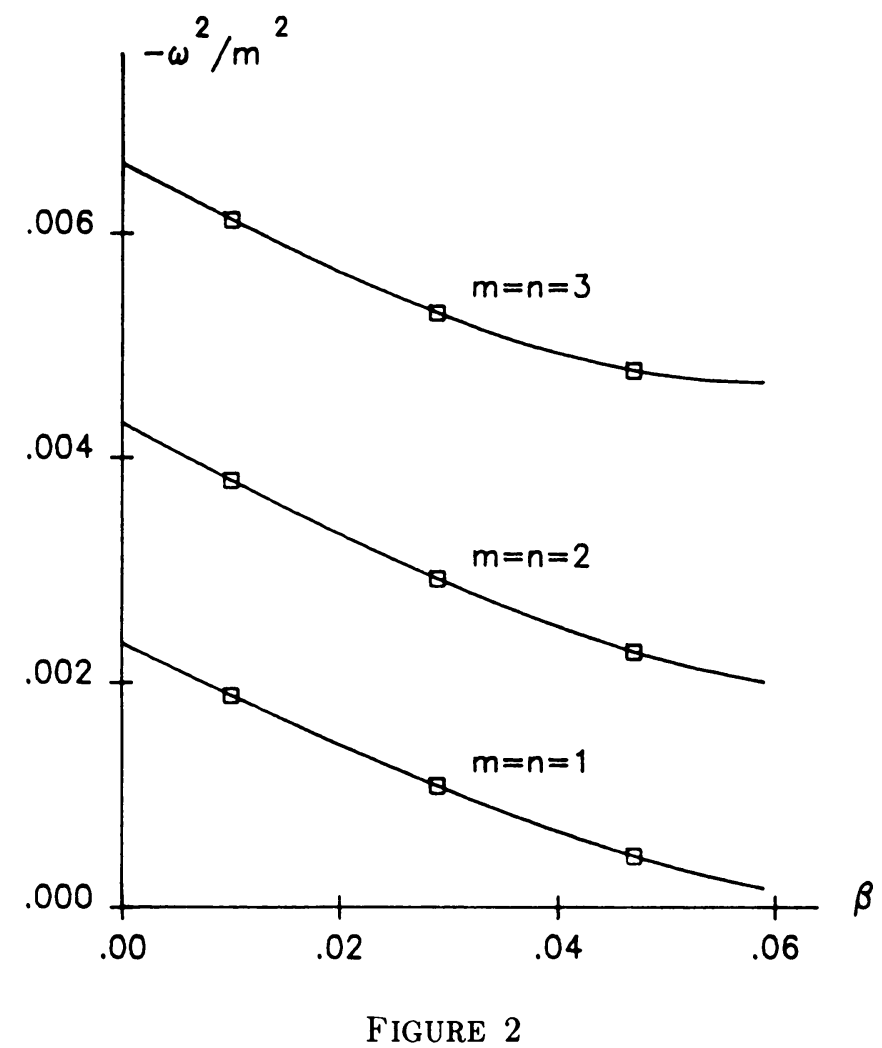

Nonlinear stability of resonant modes for a simplified Helias configuration with just the two harmonics $l=-1$ and $l=2$. A positive helical excursion of the magnetic axis results in a shift of the curves safely to the right within the stable region $-\omega^{2}>0$.

Acknowledgments. F. Bauer and K.-C. Ng developed the BETA line tracing code to generate Figure 1. This work was supported by D.O.E. Grant DE-FG0286ER53223 and N.S.F. Grant DMS-8701258.

Courant Institute of Mathematical Sciences New York University 251 Mercer Street

New York, New York 10012

1. F. BAUER, O. Betancourt \& P. GARABedian, Magnetohydrodynamic Equilibrium and Stability of Stellarators, Springer-Verlag, New York, 1984.

2. F. Bauer, O. Betancourt, P. Garabedian \& M. Wakatani, The Beta Equilibrium Stability, and Transport Codes, Academic Press, Boston, 1987.

3. K.-C. NG, Magnetic Surfaces and Neoclassical Transport in Stellarators, Ph.D. Thesis, New York University, 1987.

4. J. Nuehrenberg \& R. Zille, "Stable stellarators with medium $\beta$ and aspect ratio," Phys. Lett. $A$, v. 114, 1986, pp. 129-132. 